\title{
EXTENDED BOUSSINESQ EQUATIONS FOR WAVES IN TWO POROUS LAYERS
}

\author{
Changhoon Lee ${ }^{1}$, Van Nghi $\mathrm{Vu}^{2}$, Tae-Hwa Jung ${ }^{3}$ and Thanh Thu Huynh ${ }^{4}$ \\ In this study we continue the work of Vu et al. (2018) [Coastal Eng. 139, 85-97] to develop an \\ extended Boussinesq model that predicts the propagation of water waves in two porous layers. \\ The first and second layer can be a water layer or a porous layer. The inertial and drag \\ resistances are considered in the developed model. After being successfully validated against \\ the analytical solutions, the model is used to simulate waves propagating over a submerged \\ triangular porous bar. The numerical results show good agreement with the physical \\ experimental data of Hsiao et al. (1998) [Proc. Royal Society of London A 458, 1291-1322].
}

Keywords: two porous layers, submerged porous breakwater, extended Boussinesq equations, numerical experiment

\section{INTRODUCTION}

There exist multiple porous layers in coastal area. Submerged breakwaters are composed of two layers, i.e., upper non-porous water layer and lower porous layer. Rubble mound breakwaters are composed of two layers with different porosities. Sand beach is composed of one porous layer on the land side from the coastline. Also, it is composed of two layers with upper water and lower porous layers on the sea side. We need to have a wave model for such multiple porous layers. Until now, the Boussinesq equations have been developed for two layers with upper non-porous water layer and lower porous layer (Cruz et al., 1997; Hsiao et al., 2002). Liu and Wen (1997) developed conventional Boussinesq equations in shallow water for waves inside porous media including drag resistance but neglecting inertial resistance. These models cannot be applied for rubble mound breakwaters or sand beach. Lee et al. (2014) and $\mathrm{Vu}$ et al. (2018) developed extended Boussinesq equations for waves in porous media which considers drag and inertial resistances. The numerical solutions of their model are well verified by comparison with exact solutions and physical experiment data. In this study, we continue the work of Lee et al. and $\mathrm{Vu}$ et al. to develop a model for waves in two porous layers which can solve the aforementioned problems. The validity of the developed model is examined by comparing numerical results with analytical solution and experimental data.

\section{DEVELOPMENT OF GOVERNING EQUATIONS}

\section{Setting-up boundary value problem}

In order to develop the extended Boussinesq equations for waves inside porous layers we first set up boundary value problems for waves inside multiple porous layers. The whole domain is vertically divided into the 1 st, $2 \mathrm{nd}, \ldots$, and $j-$ th layers which are numbered from the top to the bottom layers with different porosities, as shown in Fig. 1. The free surface is located at the 1st layer.

Since the porosity is uniform, the continuity equation inside the $j-t h$ permeable layer is given by

$$
\nabla_{3} \cdot \mathbf{U}_{j}=0
$$

where $\mathbf{U}=(u, v, w)$ is the seepage velocity vector, $\nabla_{3} \equiv(\partial / \partial x, \partial / \partial y, \partial / \partial z)$ is the three-dimensional gradient operator, and the subscript $j$ implies the $j$-th layer. The momentum equation inside the $j-t h$ permeable layer is given by

\footnotetext{
${ }^{1}$ Dept. of Civil \& Environmental Engineering, Sejong University, Seoul 143-747 Korea.

${ }^{2}$ Faculty of Transportation Engineering, HoChiMinh City University of Transport, 2 Vo Oanh, Ward 25, Binh Thanh Dist., HCMC, Vietnam.

${ }^{3}$ Dept. of Civil \& Environmental Engineering, Hanbat National University, Daejeon 305-719, Korea.

${ }^{4}$ Dept. of Civil \& Environmental Engineering, Sejong University, Seoul 143-747 Korea.
} 


$$
\frac{d \mathbf{U}_{j}}{d t}+\frac{1}{\rho} \nabla_{3}\left(p_{j}+\rho g z\right)+D_{j}+I_{j}=0
$$

where $p_{j}$ is the pore pressure, $D_{j}$ is the permeable drag resistance term, and $I_{j}$ is the inertial resistance term. It should be noted that the continuity and momentum equations (1) and (2), respectively, are expressed in terms of the seepage velocity of the pore water.

Several people defined the drag resistance term differently. Ergun (1952) define the drag resistance term in the Forchheimer (1901) type using a volume-averaged discharge velocity $\mathbf{U}_{j}{ }^{\prime}\left(=\lambda_{j} \mathbf{U}_{j}\right)$. In our study, we use Ergun's definition of $D$ in terms of the seepage velocity instead as

$$
\begin{aligned}
D_{j} & \equiv\left[\alpha_{l}\left(\frac{1-\lambda}{\lambda}\right)^{2} \frac{v}{d^{2}} \frac{\mathbf{U}^{\prime}}{\lambda}+\alpha_{t} \frac{1-\lambda}{\lambda} \frac{1}{d}\left|\frac{\mathbf{U}^{\prime}}{\lambda}\right| \frac{\mathbf{U}^{\prime}}{\lambda}\right]_{j} \\
& =\left[\alpha_{l}\left(\frac{1-\lambda}{\lambda}\right)^{2} \frac{v}{d^{2}} \mathbf{U}+\alpha_{t} \frac{1-\lambda}{\lambda} \frac{1}{d}|\mathbf{U}| \mathbf{U}\right]_{j}
\end{aligned}
$$

where $\alpha_{l}$ and $\alpha_{t}$ are coefficients which represent the laminar and turbulent flow resistances, respectively, $v$ is the kinematic viscosity of water, and $d$ is the solid size. The mathematical form of Eq. (3) including the parameters $\lambda, v$, and $d$ can be derived from the Navier-Stokes equation (Irmay, 1958) or from the Reynolds equation (Burcharth and Andersen, 1995). The inertial resistance term $I_{j}$ is given by

$$
I_{j} \equiv\left[(1-\lambda)(1+\kappa) \frac{d \mathbf{U}}{d t}\right]_{j}
$$

where $\lambda$ is porosity, and $\kappa$ is added mass coefficient. In unsteady flow, the inertial resistance term is necessary to consider the divergence and convergence of streamlines in the presence of the solid material. The resistance term should consider both the local and convective accelerations. In Eq. (4), the value unity which is added to the added mass coefficient is to consider the inertial resistance of the water with the volume of solid material, and the added mass coefficient is to consider the inertial resistance in view of geometrical smoothness of the solid material. When the layer is filled with water, then $\lambda=1$ and the inertial resistance term becomes zero. When the layer is fully filled with the solid material, then $\lambda=0$ and the inertial resistance term becomes infinitely large.

Several people proposed different momentum equations including the drag and inertial resistance terms. Sollitt and Cross' (1972) momentum equation is the same as the present momentum equation (2) except that the convective acceleration is neglected to get a linear solution. van Gent's (1994) momentum equation is the same as the present momentum equation (2). However, he defined the inertial resistance term only in the local acceleration as

$$
I_{j} \equiv \frac{1-\lambda_{j}}{\lambda_{j}} \kappa_{j} \frac{\partial \mathbf{U}_{j}}{\partial t}
$$

where the value unity, which is omitted, should be added to the added mass coefficient. Cruz et al. (1997) proposed the momentum equation as

$$
\lambda_{j} \frac{d \mathbf{U}_{j}}{d t}+\frac{1}{\rho} \nabla_{3}\left(p_{j}+\rho g z\right)+D_{j}+I_{j}=0
$$

where the drag resistance term is defined in terms of the seepage velocity as

$$
D_{j} \equiv\left(\alpha_{l}^{\prime} \mathbf{U}+\alpha_{t}^{\prime}|\mathbf{U}| \mathbf{U}\right)_{j}
$$

where $\alpha_{l}^{\prime}$ and $\alpha_{t}^{\prime}$ are coefficients which represent the laminar and turbulent flow resistances, respectively, and the inertial resistance term is defined as 


$$
I_{j} \equiv\left(1-\lambda_{j}\right)\left(1+\kappa_{j}\right) \frac{d \mathbf{U}_{j}}{d t}
$$

The pressure $p_{j}$ in Eq. (6) is a volume-averaged pore pressure because the spatial variation of the pressure is related to the acceleration of the volume-averaged discharge velocity $\lambda_{j} d \mathbf{U}_{j} / d t$. Substitution of Eqs. (3) and (4) into the present momentum equation (2) gives

$$
\beta_{j} \frac{d \mathbf{U}_{j}}{d t}+\frac{1}{\rho} \nabla_{3}\left(p_{j}+\rho g z\right)+\alpha_{j} \mathbf{U}_{j}=0
$$

where $\beta_{j}$ is the inertial coefficient given by

$$
\beta_{j} \equiv[1+(1-\lambda) \kappa]_{j}
$$

and $\alpha_{j}$ is the porous resistance coefficient given by

$$
\alpha_{j}=\left[\alpha_{l}\left(\frac{1-\lambda}{\lambda}\right)^{2} \frac{v}{d^{2}}+\alpha_{t} \frac{1-\lambda}{\lambda} \frac{1}{d}|\mathbf{U}|\right]_{j}
$$

At the free surface, the dynamic and kinematic boundary conditions are given by

$$
\begin{gathered}
p_{1}=0, \quad z=\eta \\
w_{1}=\frac{\partial \eta}{\partial t}+\mathbf{u}_{1} \cdot \nabla \eta, \quad z=\eta
\end{gathered}
$$

where $\mathbf{u} \equiv(u, v)$ is the horizontal velocity vector and $\nabla \equiv(\partial / \partial x, \partial / \partial y)$ is the horizontal gradient operator. At the impermeable bottom under the lowest $J-t h$ layer, the normal velocity vanishes as

$$
\mathbf{U}_{J} \cdot \nabla_{3}\left(z+h_{J}\right)=w_{J}+\mathbf{u}_{J} \cdot \nabla h_{J}=0, \quad z=-h_{J}
$$

At the interface between the layers of different porosities, the pore pressure should be continuous in terms of the local pore pressure instead of the volume-averaged pore pressure. The difference of pressure forces between the layers will act on the solid material in the layer of smaller porosity. To the contrary, the normal flux at the interface should be continuous in terms of the volume-averaged discharge velocity. Thus, at the interface between the $j-t h$ and $(j+1)-t h$ layers, both the pore pressures at and normal fluxes through the interface are continuous as

$$
\begin{gathered}
p_{j}=p_{j+1}, \quad z=-h_{j} \\
\lambda_{j}\left(w_{j}+\mathbf{u}_{j} \cdot \nabla h_{j}\right)=\lambda_{j+1}\left(w_{j+1}+\mathbf{u}_{j+1} \cdot \nabla h_{j}\right), \quad z=-h_{j}
\end{gathered}
$$

We get the Boussinesq equations by specifying the boundary value problem with a governing equation and boundary conditions. The seepage velocity potential is defined as

$$
\mathbf{U}_{j} \equiv \nabla_{3} \Phi_{j}
$$

Then, the continuity equation (1) becomes

$$
\nabla^{2} \Phi_{j}+\frac{\partial^{2} \Phi_{j}}{\partial z^{2}}=0, \quad-h_{j}<z<\eta
$$

At the free surface, the dynamic and kinematic boundary conditions (12) and (13) become, respectively,

$$
\beta_{1}\left[\frac{\partial \Phi_{1}}{\partial t}+\frac{1}{2}\left(\nabla_{3} \Phi_{1}\right)^{2}\right]+g \eta+\alpha_{1} \Phi_{1}=0, \quad z=\eta
$$




$$
\frac{\partial \Phi_{1}}{\partial z}=\frac{\partial \eta}{\partial t}+\nabla \Phi_{1} \cdot \nabla \eta, \quad z=\eta
$$

At the bottom, the kinematic boundary condition (14) becomes

$$
\frac{\partial \Phi_{J}}{\partial z}=-\nabla \Phi_{J} \cdot \nabla h_{J}, \quad z=-h_{J}
$$

At the interface, the continuities of the pore pressure and normal flux (15) and (16) become, respectively,

$$
\begin{gathered}
\beta_{j}\left[\frac{\partial \Phi_{j}}{\partial t}+\frac{1}{2}\left(\nabla_{3} \Phi_{j}\right)^{2}\right]+\alpha_{j} \Phi_{j} \\
=\beta_{j+1}\left[\frac{\partial \Phi_{j+1}}{\partial t}+\frac{1}{2}\left(\nabla_{3} \Phi_{j+1}\right)^{2}\right]+\alpha_{j+1} \Phi_{j+1}, \quad z=-h_{j} \\
\lambda_{j}\left(\frac{\partial \Phi_{j}}{\partial z}+\nabla \Phi_{j} \cdot \nabla h_{j}\right)=\lambda_{j+1}\left(\frac{\partial \Phi_{j+1}}{\partial z}+\nabla \Phi_{j+1} \cdot \nabla h_{j+1}\right), \quad z=-h_{j}
\end{gathered}
$$

The variables are normalized using the relevant characteristic length and time as

$$
\begin{aligned}
& x^{\prime}=\frac{x}{l}, \quad y^{\prime}=\frac{y}{l}, \quad z^{\prime}=\frac{z}{h_{0}}, \quad h^{\prime}=\frac{h}{h_{0}}, \quad \eta^{\prime}=\frac{\eta}{a}, \quad t^{\prime}=\frac{\sqrt{g h_{0}}}{l} t, \\
& \Phi^{\prime}=\frac{h_{0}}{a l \sqrt{g h_{0}}} \Phi, \quad u^{\prime}=\frac{h_{0}}{a \sqrt{g h_{0}}} u, \quad \alpha^{\prime}=\frac{l}{\sqrt{g h_{0}}} \alpha
\end{aligned}
$$

where $l$ is the wavelength, $h_{0}$ is the maximum water depth, $a$ is the maximum amplitude of the water surface elevation, $t$ is the wave period. When the normalization is applied, the terms in the governing equation and boundary conditions will group according to two non-dimensional quantities

$$
\varepsilon=\frac{a}{h_{0}}, \quad \mu=\frac{h_{0}}{l}
$$

where $\varepsilon$ is the nonlinearity parameter and $\mu$ is the dispersivity parameter. Omitting the primes for convenience, the continuity equation (18) and boundary conditions (19)-(23) become, respectively,

$$
\begin{gathered}
\mu^{2} \nabla^{2} \Phi_{j}+\frac{\partial^{2} \Phi_{j}}{\partial z^{2}}=0, \quad-1<z<\varepsilon \eta \\
\mu^{2}\left(\beta_{1} \frac{\partial \Phi_{1}}{\partial t}+\alpha_{1} \Phi_{1}+\eta\right)+\varepsilon \beta_{1} \frac{1}{2}\left[\mu^{2}\left(\nabla \Phi_{1}\right)^{2}+\left(\frac{\partial \Phi_{1}}{\partial z}\right)^{2}\right]=0, \quad z=\varepsilon \eta \\
\frac{\partial \Phi_{1}}{\partial z}=\mu^{2}\left(\frac{\partial \eta}{\partial t}+\varepsilon \nabla \Phi_{1} \cdot \nabla \eta\right), \quad z=\varepsilon \eta \\
\frac{\partial \Phi_{J}}{\partial z}=-\mu^{2} \nabla \Phi_{J} \cdot \nabla h_{J}, \quad z=-h_{J}
\end{gathered}
$$




$$
\begin{aligned}
& \mu^{2}\left(\beta_{j} \frac{\partial}{\partial t}+\alpha_{j}\right) \Phi_{j}+\varepsilon \beta_{j} \frac{1}{2}\left[\left(\frac{\partial \Phi_{j}}{\partial z}\right)^{2}+\mu^{2}\left(\nabla \Phi_{j}\right)^{2}\right] \\
& =\mu^{2}\left(\beta_{j+1} \frac{\partial}{\partial t}+\alpha_{j+1}\right) \Phi_{j+1}+\varepsilon \beta_{j+1} \frac{1}{2}\left[\left(\frac{\partial \Phi_{j+1}}{\partial z}\right)^{2}+\mu^{2}\left(\nabla \Phi_{j+1}\right)^{2}\right], \quad z=-h_{j} \\
& \lambda_{j}\left(\frac{\partial \Phi_{j}}{\partial z}+\mu^{2} \nabla \Phi_{j} \cdot \nabla h_{j}\right)=\lambda_{j+1}\left(\frac{\partial \Phi_{j+1}}{\partial z}+\mu^{2} \nabla \Phi_{j+1} \cdot \nabla h_{j+1}\right), \quad z=-h_{j}
\end{aligned}
$$

The velocity potential can be expressed as a power series in the vertical coordinate given by

$$
\Phi_{j}(x, y, z, t)=\sum_{n=0}^{\infty}\left[z+h_{j}(x, y)\right]^{n} \varphi_{j, n}(x, y, t)
$$

Then, we have

$$
\begin{gathered}
\nabla \Phi_{j}=\sum_{n=0}^{\infty}\left(z+h_{j}\right)^{n} \nabla \varphi_{j, n}+\sum_{n=0}^{\infty}\left(z+h_{j}\right)^{n}(n+1)\left(\nabla h_{j}\right) \varphi_{j, n+1} \\
\nabla^{2} \Phi_{j}=\sum_{n=0}^{\infty}\left(z+h_{j}\right)^{n} \nabla^{2} \varphi_{j, n}+\sum_{n=0}^{\infty}\left(z+h_{j}\right)^{n}(n+1)\left[2 \nabla h_{j} \cdot \nabla \varphi_{j, n+1}+\nabla^{2} h_{j} \varphi_{j, n+1}\right] \\
+\sum_{n=0}^{\infty}\left(z+h_{j}\right)^{n}(n+1)(n+2)\left(\nabla h_{j}\right)^{2} \varphi_{j, n+2} \\
\frac{\partial \Phi_{j}}{\partial z}=\sum_{n=0}^{\infty}\left(z+h_{j}\right)^{n}(n+1) \varphi_{j, n+1} \\
\frac{\partial^{2} \Phi_{j}}{\partial z^{2}}=\sum_{n=0}^{\infty}\left(z+h_{j}\right)^{n}(n+1)(n+2) \varphi_{j, n+2}
\end{gathered}
$$

Substitution of Eqs. (34) and (36) into the continuity equation (26) gives

$$
\begin{aligned}
& (n+1)(n+2)\left[1+\mu^{2}\left(\nabla h_{j}\right)^{2}\right] \varphi_{j, n+2} \\
& +(n+1) \mu^{2}\left[2 \nabla h_{j} \cdot \nabla \varphi_{j, n+1}+\nabla^{2} h_{j} \varphi_{j, n+1}\right]+\mu^{2} \nabla^{2} \varphi_{j, n}=0, \quad n=0,1,2, \mathrm{~L}
\end{aligned}
$$

Substitution of Eqs. (33) and (35) into the bottom boundary condition (29) gives

$$
\varphi_{J, 1}=-\mu^{2} \frac{\nabla h_{J} \cdot \nabla \varphi_{J, 0}}{1+\mu^{2}\left(\nabla h_{J}\right)^{2}}
$$

And, the velocity potential functions in the lowest layer $\varphi_{I, 2}, \varphi_{I, 3}, \cdots$ can be expressed in terms of $\varphi_{J, 0}$ using Eqs. (37) and (38) as

$$
\begin{gathered}
\varphi_{J, 2}=-\frac{\mu^{2}}{2\left[1+\mu^{2}\left(\nabla h_{J}\right)^{2}\right]} \nabla^{2} \varphi_{J, 0}+\mu^{4} \nabla \cdot\left[\nabla h_{J}\left(\nabla h_{J} \cdot \nabla \varphi_{J, 0}\right)\right]+O\left(\mu^{6}\right) \\
\varphi_{J, 3}=\frac{\mu^{4}}{6}\left[2 \nabla h_{J} \cdot \nabla\left(\nabla^{2} \varphi_{J, 0}\right)+\nabla^{2} h_{J} \nabla^{2} \varphi_{J, 0}+\nabla^{2}\left(\nabla h_{J} \cdot \nabla \varphi_{J, 0}\right)\right]+O\left(\mu^{6}\right)
\end{gathered}
$$

Thus, the velocity potential in the bottom layer $\Phi_{J}$ can be expressed to the order of $O\left(\mu^{2}\right)$ as 


$$
\Phi_{J}=\varphi_{J, 0}-\frac{\mu^{2}}{2}\left[2\left(z+h_{J}\right) \nabla h_{J} \cdot \nabla \varphi_{J, 0}+\left(z+h_{J}\right)^{2} \nabla^{2} \varphi_{J, 0}\right]+O\left(\mu^{4}\right)
$$

\section{Development of Boussinesq equations for waves in two porous layers}

For two permeable layers, all the subscripts $j$ in the variables are $j=1,2$. Thus, from Eq. (41), the velocity potential in the bottom layer $\Phi_{2}$ becomes

$$
\Phi_{2}=\varphi_{2,0}-\frac{\mu^{2}}{2}\left[2\left(z+h_{2}\right) \nabla h_{2} \cdot \nabla \varphi_{2,0}+\left(z+h_{2}\right)^{2} \nabla^{2} \varphi_{2,0}\right]+O\left(\mu^{4}\right)
$$

The velocity potentials $\Phi_{1}$ and $\Phi_{2}$ are related through the interface boundary conditions (30) or (31). We obtain the velocity potential function in the 1 st layer $\varphi_{1,1}$ by applying the interface boundary condition (31) with $j=1$ and using Eq. (42) as

$$
\varphi_{1,1}=-\mu^{2}\left\{\nabla h_{1} \cdot \nabla \varphi_{1,0}+\frac{\lambda_{2}}{\lambda_{1}} \nabla \cdot\left[\left(h_{2}-h_{1}\right) \nabla \varphi_{2,0}\right]\right\}+O\left(\mu^{4}\right)
$$

And, the velocity potential functions in the 1st layer $\varphi_{1,2}, \varphi_{1,3}, \ldots$ can be expressed in terms of $\varphi_{1,0}$ and $\varphi_{2,0}$ using Eqs. (37) and (43) as

$$
\begin{gathered}
\varphi_{1,2}=-\frac{\mu^{2}}{2} \nabla^{2} \varphi_{1,0}+O\left(\mu^{4}\right) \\
\varphi_{1,3}=O\left(\mu^{4}\right)
\end{gathered}
$$

Thus, the velocity potential in the $1^{\text {st }}$ layer $\Phi_{1}$ can be expressed to the order of $O\left(\mu^{2}\right)$ as

$$
\Phi_{1}=\varphi_{1,0}-\frac{\mu^{2}}{2}\left\langle 2\left(z+h_{1}\right)\left\{\nabla h_{1} \nabla \varphi_{1,0}+\frac{\lambda_{2}}{\lambda_{1}} \nabla \cdot\left[\left(h_{2}-h_{1}\right) \nabla \varphi_{2,0}\right]\right\}+\left(z+h_{1}\right)^{2} \nabla^{2} \varphi_{1,0}\right\rangle+O\left(\mu^{4}\right)
$$

The momentum equation in the $1^{\text {st }}$ layer is obtained by substituting Eq. (46) into the dynamic freesurface boundary condition (27) and then applying $\nabla$ to the resulting equation as

$$
\begin{aligned}
& \left(\beta_{1} \frac{\partial}{\partial t}+\alpha_{1}\right) \mathbf{u}_{1,0}+\nabla \eta+\varepsilon \beta_{1} \mathbf{u}_{1,0} \cdot \nabla \mathbf{u}_{1,0} \\
& -\frac{\mu^{2}}{2}\left(\beta_{1} \frac{\partial}{\partial t}+\alpha_{1}\right) \nabla\left\{h_{1}^{2} \nabla \cdot \mathbf{u}_{1,0}+2 h_{1} \nabla h_{1} \cdot \mathbf{u}_{1,0}+2 h_{1} \frac{\lambda_{2}}{\lambda_{1}} \nabla \cdot\left[\left(h_{2}-h_{1}\right) \mathbf{u}_{2,0}\right]\right\}=\mathrm{O}\left(\varepsilon \mu^{2}, \mu^{4}\right)
\end{aligned}
$$

where $\mathbf{u}_{2,0} \equiv \nabla \varphi_{2,0}$ is the seepage velocity in the $2^{\text {nd }}$ layer. The momentum equation in the $2^{\text {nd }}$ layer is obtained by substituting Eqs. (42) and (46) into the interface continuity of pore pressure (30) with $j=1$, and then applying $\nabla$ to the resulting equation as

$$
\begin{aligned}
& \left(\beta_{1} \frac{\partial}{\partial t}+\alpha_{1}\right) \mathbf{u}_{1,0}+\varepsilon \beta_{1} \mathbf{u}_{1,0} \cdot \nabla \mathbf{u}_{1,0} \\
& =\left(\beta_{2} \frac{\partial}{\partial t}+\alpha_{2}\right)\left\{\mathbf{u}_{2,0}-\frac{\mu^{2}}{2} \nabla\left[2\left(h_{2}-h_{1}\right) \nabla h_{2} \cdot \mathbf{u}_{2,0}+\left(h_{2}-h_{1}\right)^{2} \nabla \cdot \mathbf{u}_{2,0}\right]\right\}+\varepsilon \beta_{2} \mathbf{u}_{2,0} \cdot \nabla \mathbf{u}_{2,0}+\mathrm{O}\left(\varepsilon \mu^{2}, \mu^{4}\right)
\end{aligned}
$$

Or, using Eq. (47), 


$$
\begin{aligned}
& \left(\beta_{2} \frac{\partial}{\partial t}+\alpha_{2}\right) \mathbf{u}_{2,0}+\nabla \eta+\varepsilon \beta_{2} \mathbf{u}_{2,0} \cdot \nabla \mathbf{u}_{2,0} \\
& -\frac{\mu^{2}}{2}\left(\beta_{2} \frac{\partial}{\partial t}+\alpha_{2}\right) \nabla\left[2\left(h_{2}-h_{1}\right) \nabla h_{2} \cdot \mathbf{u}_{2,0}+\left(h_{2}-h_{1}\right)^{2} \nabla \cdot \mathbf{u}_{2,0}\right] \\
& -\frac{\mu^{2}}{2}\left(\beta_{1} \frac{\partial}{\partial t}+\alpha_{1}\right) \nabla\left\langle 2 h_{1}\left\{\nabla h_{1} \cdot \mathbf{u}_{1,0}+\frac{\lambda_{2}}{\lambda_{1}} \nabla \cdot\left[\left(h_{2}-h_{1}\right)\right] \mathbf{u}_{2,0}\right\}+h_{1}^{2} \nabla \cdot \mathbf{u}_{1,0}\right\rangle=\mathrm{O}\left(\varepsilon \mu^{2}, \mu^{4}\right)
\end{aligned}
$$

Here, we use the depth-averaged velocity defined as

$$
\overline{\mathbf{u}}_{j} \equiv \begin{cases}\frac{1}{h_{1}+\varepsilon \eta} \int_{-h_{1}}^{\varepsilon \eta} \nabla \Phi_{1} d z, & j=1 \\ \frac{1}{h_{2}-h_{1}} \int_{-h_{2}}^{-h_{1}} \nabla \Phi_{2} d z, & j=2\end{cases}
$$

After substituting Eq. (46) into Eq. (50) with $j=1$, the seepage velocity at the lower boundary of the $1^{\text {st }}$ layer can be expressed in terms of the depth-averaged velocity as

$$
\begin{aligned}
\mathbf{u}_{1,0}= & \overline{\mathbf{u}}_{1}+\frac{\mu^{2}}{2}\left[\frac{h_{1}^{2}}{3} \nabla\left(\nabla \cdot \overline{\mathbf{u}}_{1}\right)+h_{1} \nabla\left(\nabla h_{1} \cdot \overline{\mathbf{u}}_{1}\right)+h_{1} \nabla h_{1} \nabla \cdot \overline{\mathbf{u}}_{1}+2 \nabla h_{1} \nabla h_{1} \cdot \overline{\mathbf{u}}_{1}\right] \\
& +\frac{\mu^{2}}{2}\left\langle h_{1} \nabla\left\{\frac{\lambda_{2}}{\lambda_{1}} \nabla \cdot\left[\left(h_{2}-h_{1}\right) \overline{\mathbf{u}}_{2}\right]+2 \nabla h_{1} \frac{\lambda_{2}}{\lambda_{1}} \nabla \cdot\left[\left(h_{2}-h_{1}\right) \overline{\mathbf{u}}_{2}\right]\right\}\right\rangle+\mathrm{O}\left(\mu^{4}\right)
\end{aligned}
$$

After substituting Eq. (42) into Eq. (50) with $j=2$, the seepage velocity at the lower boundary of the $2^{\text {nd }}$ layer can be expressed in terms of the depth-averaged velocity as

$$
\begin{aligned}
\mathbf{u}_{2,0}= & \overline{\mathbf{u}}_{2}+\frac{\mu^{2}}{2}\left[\frac{\left(h_{2}-h_{1}\right)^{2}}{3} \nabla\left(\nabla \cdot \overline{\mathbf{u}}_{2}\right)+\left(h_{2}-h_{1}\right) \nabla\left(\nabla h_{2} \cdot \overline{\mathbf{u}}_{2}\right)+\left(h_{2}-h_{1}\right) \nabla h_{2} \nabla \cdot \overline{\mathbf{u}}_{2}+2 \nabla h_{2} \nabla h_{2} \cdot \overline{\mathbf{u}}_{2}\right] \\
& +\mathrm{O}\left(\mu^{4}\right)
\end{aligned}
$$

Substitution of Eqs. (51) and (52) into Eq. (47) gives the momentum equation in the 1st layer as

$$
\begin{aligned}
& \left(\beta_{1} \frac{\partial}{\partial t}+\alpha_{1}\right) \overline{\mathbf{u}}_{1}+\nabla \eta+\varepsilon \beta_{1} \overline{\mathbf{u}}_{1} \cdot \nabla \overline{\mathbf{u}}_{1} \\
& +\frac{\mu^{2}}{2}\left(\beta_{1} \frac{\partial}{\partial t}+\alpha_{1}\right)\left\langle\frac{h_{1}^{2}}{3} \nabla\left(\nabla \cdot \overline{\mathbf{u}}_{1}\right)-h_{1} \nabla\left[\nabla \cdot\left(h_{1} \overline{\mathbf{u}}_{1}\right)\right]-h_{1} \nabla\left\{\frac{\lambda_{2}}{\lambda_{1}} \nabla \cdot\left[\left(h_{2}-h_{1}\right) \overline{\mathbf{u}}_{2}\right]\right\}\right)=\mathrm{O}\left(\varepsilon \mu^{2}, \mu^{4}\right)
\end{aligned}
$$

Substitution of Eqs. (51) and (52) into Eq. (49) gives the momentum equation in the 2nd layer as

$$
\begin{aligned}
& \left(\beta_{2} \frac{\partial}{\partial t}+\alpha_{2}\right) \overline{\mathbf{u}}_{2}+\nabla \eta+\varepsilon \beta_{2} \overline{\mathbf{u}}_{2} \cdot \nabla \overline{\mathbf{u}}_{2}+\frac{\mu^{2}}{2}\left(\beta_{2} \frac{\partial}{\partial t}+\alpha_{2}\right) \times \\
& {\left[-\frac{2}{3}\left(h_{2}-h_{1}\right)^{2} \nabla\left(\nabla \cdot \overline{\mathbf{u}}_{2}\right)-\left(h_{2}-h_{1}\right) \nabla\left(\nabla h_{2} \cdot \overline{\mathbf{u}}_{2}\right)-\left(h_{2}-h_{1}\right) \nabla\left(h_{2}-2 h_{1}\right) \nabla \cdot \overline{\mathbf{u}}_{2}+2 \nabla h_{1} \nabla h_{2} \cdot \overline{\mathbf{u}}_{2}\right]} \\
& -\frac{\mu^{2}}{2}\left(\beta_{1} \frac{\partial}{\partial t}+\alpha_{1}\right) \nabla\left\{\nabla \cdot\left(h_{1}^{2} \overline{\mathbf{u}}_{1}\right)+2 h_{1} \frac{\lambda_{2}}{\lambda_{1}} \nabla \cdot\left[\left(h_{2}-h_{1}\right) \overline{\mathbf{u}}_{2}\right]\right\}=\mathrm{O}\left(\varepsilon \mu^{2}, \mu^{4}\right)
\end{aligned}
$$

The continuity equation is obtained by substituting Eq. (46) into the kinematic free-surface boundary condition (28) as

$$
\frac{\partial \eta}{\partial t}+\nabla \cdot\left[\left(h_{1}+\varepsilon \eta\right) \overline{\mathbf{u}}_{1}\right]+\frac{\lambda_{2}}{\lambda_{1}} \nabla \cdot\left[\left(h_{2}-h_{1}\right) \overline{\mathbf{u}}_{2}\right]=0
$$


Eqs. (53)-(55) are the set of Boussinesq equations for waves in two permeable layers.

If the 1st layer is not in porous media but in clean water, then $\lambda_{1}=1, \beta_{1}=1$ and $\alpha_{1}=0$, and thus Eqs. (53)-(55) become the Boussinesq equations of Cruz et al. (1997) which are for waves on a single permeable layer. If the 1st layer is in clean water and the interface between the 1st and 2nd layers is rough, then $\lambda_{1}=1, \beta_{1}=1$ and $\lambda_{1}=1, \alpha_{1}=f|\mathbf{U}| / 2$ where $f$ is the friction factor related to the shear stress on the rough interface. Further, if waves are very long, the resulting equations (53)-(55) are similar to the shallow-water equations of Kobayashi (1986).

In physical variables, Eqs. (53)-(55) are

$$
\begin{aligned}
& \left(\beta_{1} \frac{\partial}{\partial t}+\alpha_{1}\right) \overline{\mathbf{u}}_{1}+g \nabla \eta+\beta_{1} \overline{\mathbf{u}}_{1} \cdot \nabla \overline{\mathbf{u}}_{1} \\
& +\frac{1}{2}\left(\beta_{1} \frac{\partial}{\partial t}+\alpha_{1}\right)\left\langle\frac{h_{1}^{2}}{3} \nabla\left(\nabla \cdot \overline{\mathbf{u}}_{1}\right)-h_{1} \nabla\left[\nabla \cdot\left(h_{1} \overline{\mathbf{u}}_{1}\right)\right]-h_{1} \nabla\left\{\frac{\lambda_{2}}{\lambda_{1}} \nabla \cdot\left[\left(h_{2}-h_{1}\right) \overline{\mathbf{u}}_{2}\right]\right\}\right)=0 \\
& \left(\beta_{2} \frac{\partial}{\partial t}+\alpha_{2}\right) \overline{\mathbf{u}}_{2}+g \nabla \eta+\beta_{2} \overline{\mathbf{u}}_{2} \cdot \nabla \overline{\mathbf{u}}_{2}+\frac{1}{2}\left(\beta_{2} \frac{\partial}{\partial t}+\alpha_{2}\right) \times \\
& {\left[-\frac{2}{3}\left(h_{2}-h_{1}\right)^{2} \nabla\left(\nabla \cdot \overline{\mathbf{u}}_{2}\right)-\left(h_{2}-h_{1}\right) \nabla\left(\nabla h_{2} \cdot \overline{\mathbf{u}}_{2}\right)-\left(h_{2}-h_{1}\right) \nabla\left(h_{2}-2 h_{1}\right) \nabla \cdot \overline{\mathbf{u}}_{2}+2 \nabla h_{1} \nabla h_{2} \cdot \overline{\mathbf{u}}_{2}\right]} \\
& -\frac{1}{2}\left(\beta_{1} \frac{\partial}{\partial t}+\alpha_{1}\right) \nabla\left\{\nabla \cdot\left(h_{1}^{2} \overline{\mathbf{u}}_{1}\right)+2 h_{1} \frac{\lambda_{2}}{\lambda_{1}} \nabla \cdot\left[\left(h_{2}-h_{1}\right) \overline{\mathbf{u}}_{2}\right]\right\}=0 \\
& \frac{\partial \eta}{\partial t}+\nabla \cdot\left[\left(h_{1}+\eta\right) \overline{\mathbf{u}}_{1}\right]+\frac{\lambda_{2}}{\lambda_{1}} \nabla \cdot\left[\left(h_{2}-h_{1}\right) \overline{\mathbf{u}}_{2}\right]=0
\end{aligned}
$$

\section{Extended Boussinesq equations for waves inside two permeable layers}

The momentum equation (56) is rewritten by expanding the dispersive term as

$$
\begin{aligned}
& \left(\beta_{1} \frac{\partial}{\partial t}+\alpha_{1}\right) \overline{\mathbf{u}}_{1}+g \nabla \eta+\beta_{1} \overline{\mathbf{u}}_{1} \cdot \nabla \overline{\mathbf{u}}_{1} \\
& +\frac{1}{2}\left(\beta_{1} \frac{\partial}{\partial t}+\alpha_{1}\right)\left\langle\frac{h_{1}^{2}}{3} \nabla\left(\nabla \cdot \overline{\mathbf{u}}_{1}\right)-h_{1} \nabla\left\{\frac{\lambda_{2}}{\lambda_{1}} \nabla \cdot\left[\left(h_{2}-h_{1}\right) \overline{\mathbf{u}}_{2}\right]\right\}\right\rangle \\
& -\left(\frac{1}{2}+\gamma_{1}\right)\left(\beta_{1} \frac{\partial}{\partial t}+\alpha_{1}\right) h_{1} \nabla\left[\nabla \cdot\left(h_{1} \overline{\mathbf{u}}_{1}\right)\right]-\gamma_{1} g h_{1} \nabla\left[\nabla \cdot\left(h_{1} \nabla \eta\right)\right]=0
\end{aligned}
$$

From the lowest-order momentum equation in the $2^{\text {nd }}$ layer, we can get the following relation

$$
\frac{\partial \overline{\mathbf{u}}_{2}}{\partial t}=-\frac{1}{\beta_{2}}\left(\alpha_{2} \overline{\mathbf{u}}_{2}+g \nabla \eta\right)
$$

Using this relation, the last term in the momentum equation (59) becomes

$$
\begin{aligned}
& \nabla\left\{h_{1} \frac{\lambda_{2}}{\lambda_{1}} \nabla \cdot\left[\left(h_{2}-h_{1}\right)\left(\beta_{1} \frac{\partial}{\partial t}+\alpha_{1}\right) \overline{\mathbf{u}}_{2}\right]\right\} \\
& =\nabla\left\langle h_{1} \frac{\lambda_{2}}{\lambda_{1}} \nabla \cdot\left\{\left(h_{2}-h_{1}\right)\left[\left(\alpha_{1}-\alpha_{2} \frac{\beta_{1}}{\beta_{2}}\right) \overline{\mathbf{u}}_{2}-g \frac{\beta_{1}}{\beta_{2}} \nabla \eta\right]\right\}\right\rangle
\end{aligned}
$$

From the above equation, the zero equation is obtained by multiplying a small number $-\gamma_{2}$ as 


$$
\begin{aligned}
& -\gamma_{2}\left\langle\left(\beta_{1} \frac{\partial}{\partial t}+\alpha_{1}\right) \nabla\left\{h_{1} \frac{\lambda_{2}}{\lambda_{1}} \nabla \cdot\left[\left(h_{2}-h_{1}\right) \overline{\mathbf{u}}_{2}\right]\right\}+\left(\alpha_{2} \frac{\beta_{1}}{\beta_{2}}-\alpha_{1}\right) \nabla\left\{h_{1} \frac{\lambda_{2}}{\lambda_{1}} \nabla \cdot\left[\left(h_{2}-h_{1}\right) \overline{\mathbf{u}}_{2}\right]\right\}\right. \\
& \left.+g \frac{\beta_{1}}{\beta_{2}} \nabla\left\{h_{1} \frac{\lambda_{2}}{\lambda_{1}} \nabla \cdot\left[\left(h_{2}-h_{1}\right) \nabla \eta\right]\right\}\right\}=0
\end{aligned}
$$

When this is added to Eq. (59), we have the extended momentum equation

$$
\begin{aligned}
& \left(\beta_{2} \frac{\partial}{\partial t}+\alpha_{2}\right) \overline{\mathbf{u}}_{2}+g \nabla \eta+\beta_{2} \overline{\mathbf{u}}_{2} \cdot \nabla \overline{\mathbf{u}}_{2}+\frac{1}{2}\left(\beta_{2} \frac{\partial}{\partial t}+\alpha_{2}\right)\left[-\frac{2}{3}\left(h_{2}-h_{1}\right)^{2} \nabla\right. \\
& \left.\left(\nabla \cdot \overline{\mathbf{u}}_{2}\right)-\left(h_{2}-h_{1}\right) \nabla\left(\nabla h_{2} \cdot \overline{\mathbf{u}}_{2}\right)-\left(h_{2}-h_{1}\right) \nabla\left(h_{2}-2 h_{1}\right) \nabla \cdot \overline{\mathbf{u}}_{2}+2 \nabla h_{1} \nabla h_{2} \cdot \overline{\mathbf{u}}_{2}\right] \\
& -\frac{1}{2}\left(\beta_{1} \frac{\partial}{\partial t}+\alpha_{1}\right) \nabla\left[\nabla \cdot\left(h_{1}^{2} \overline{\mathbf{u}}_{1}\right)\right]-\left[\left(1+\gamma_{2}\right)\left(\beta_{1} \frac{\partial}{\partial t}+\alpha_{1}\right)+\gamma_{2}\left(\alpha_{2} \frac{\beta_{1}}{\beta_{2}}-\alpha_{1}\right)\right] \times \\
& \nabla\left\{h_{1} \frac{\lambda_{2}}{\lambda_{1}} \nabla \cdot\left[\left(h_{2}-h_{1}\right) \overline{\mathbf{u}}_{2}\right]\right\}-\gamma_{2} g \frac{\beta_{1}}{\beta_{2}} \nabla\left\{h_{1} \frac{\lambda_{2}}{\lambda_{1}} \nabla \cdot\left[\left(h_{2}-h_{1}\right) \nabla \eta\right]\right\}=0
\end{aligned}
$$

\section{NUMERICAL VERIFICATION}

\section{Numerical scheme}

The finite-difference method is applied to solve the extended Boussinesq equations (58), (59), and (63). The time derivative terms are discretized with the Adam-Bashforth-Moulton predictor and corrector scheme following Wei and Kirby (1995). The first-order spatial derivative terms are discretized up to $\mathrm{O}\left(\Delta x^{4}\right)$. The higher-order spatial derivative terms in the momentum equations that are dispersive terms are discretized up to $\mathrm{O}\left(\Delta x^{2}\right)$. The variables $\eta, u_{1}, u_{2}$ are placed in an un-staggered grid system. Eqs. (58), (59), and (63) can be rewritten in one-dimensional domain as

$$
\begin{aligned}
\eta_{t} & =E\left(\eta, u_{1}, u_{2}\right) \\
{\left[U_{1}\left(u_{1}, u_{2}\right)\right]_{t} } & =F_{1}\left(\eta, u_{1}, u_{2}\right)+H_{1}\left(u_{2}\right) \\
{\left[U_{2}\left(u_{1}, u_{2}\right)\right]_{t} } & =F_{2}\left(\eta, u_{1}, u_{2}\right)+H_{2}\left(u_{1}\right)
\end{aligned}
$$

where

$$
\begin{gathered}
E\left(\eta, u_{1}, u_{2}\right)=-\left[\left(h_{1}+\eta\right) u_{1}\right]_{x}-\frac{\lambda_{2}}{\lambda_{1}}\left[\left(h_{2}-h_{1}\right) u_{2}\right]_{x} \\
{\left[U_{1}\left(\mathrm{u}_{1}, \mathrm{u}_{2}\right)\right]_{t}=F_{1}\left(\eta, \mathrm{u}_{1}, \mathrm{u}_{2}\right)+\mathrm{H}_{1}\left(u_{2}\right)} \\
{\left[U_{2}\left(\mathrm{u}_{1}, \mathrm{u}_{2}\right)\right]_{t}=F_{2}\left(\eta, \mathrm{u}_{1}, \mathrm{u}_{2}\right)+\mathrm{H}_{2}\left(u_{1}\right)} \\
{\left[U_{1}\left(\mathrm{u}_{1}, \mathrm{u}_{2}\right)\right]=u_{1}+\frac{1}{6} h_{1}^{2} u_{1 x x}-\left(\frac{1}{2}+\gamma_{1}\right) h_{1}\left(h_{1} u_{1}\right)_{x x}} \\
F_{1}\left(\eta, \mathrm{u}_{1}, \mathrm{u}_{2}\right)=-\frac{\alpha_{1}}{\beta_{1}} u_{1}-u_{1} u_{1 x}-\frac{g}{\beta_{1}} \eta_{x}-\frac{1}{6} \frac{\alpha_{1}}{\beta_{1}} h_{1}^{2} u_{1 x x}+\frac{1}{2} \frac{\alpha_{1}}{\beta_{1}} \frac{\lambda_{2}}{\lambda_{1}} h_{1}\left(h_{2}-h_{1}\right) u_{2 x x} \\
+\left(\frac{1}{2}+\gamma_{1}\right) \frac{\alpha_{1}}{\beta_{1}} h_{1}\left(h_{1} u_{1}\right)_{x x}+\frac{\gamma_{1}}{\beta_{1}} g h_{1}\left(h_{1} \eta_{x}\right)_{x x}
\end{gathered}
$$




$$
\begin{gathered}
H_{1}\left(\mathrm{u}_{2}\right)=\frac{1}{2} h_{1} \frac{\lambda_{2}}{\lambda_{1}}\left[\left(h_{2}-h_{1}\right) u_{2}\right]_{x x} \\
{\left[U_{2}\left(\mathrm{u}_{1}, \mathrm{u}_{2}\right)\right]=u_{2}-\frac{1}{2}\left[\frac{2}{3}\left(h_{2}-h_{1}\right)^{2} u_{2 x x}\right]-\frac{1}{2}\left(h_{2}-h_{1}\right) h_{2 x x} u_{2}-\frac{1}{2}\left(h_{2}-h_{1}\right) h_{2 x} u_{2 x}} \\
+\frac{1}{2}\left(h_{2}-h_{1}\right)\left(2 h_{1}-h_{2}\right)_{x} u_{2 x}+h_{1 x} h_{2 x} u_{2}-\left(1+\gamma_{2}\right) \frac{\beta_{1}}{\beta_{2}} \frac{\lambda_{2}}{\lambda_{1}} h_{1 x}\left[\left(h_{2}-h_{1}\right) u_{2}\right]_{x}-\left(1+\gamma_{2}\right) \frac{\beta_{1}}{\beta_{2}} \frac{\lambda_{2}}{\lambda_{1}} h_{1}\left[\left(h_{2}-h_{1}\right) u_{2}\right]_{x x} \\
F_{2}\left(\eta, \mathrm{u}_{1}, \mathrm{u}_{2}\right)=-\frac{\alpha_{2}}{\beta_{2}} u_{2}-u_{2} u_{2 x}-\frac{g}{\beta_{2}} \eta_{x}+\frac{1}{3} \frac{\alpha_{2}}{\beta_{2}}\left(h_{2}-h_{1}\right)^{2} u_{2 x x}+\frac{1}{2} \frac{\alpha_{2}}{\beta_{2}}\left(h_{2}-h_{1}\right) h_{2 x x} u_{2} \\
+\frac{1}{2} \frac{\alpha_{2}}{\beta_{2}}\left(h_{2}-h_{1}\right) h_{2 x} u_{2 x}-\frac{1}{2} \frac{\alpha_{2}}{\beta_{2}}\left(h_{2}-h_{1}\right)\left(2 h_{1}-h_{2}\right)_{x} u_{2 x}-\frac{\alpha_{2}}{\beta_{2}} h_{1 x} h_{2 x} u_{2}+\left(1+\gamma_{2}\right) \frac{\alpha_{1}}{\beta_{2}} \frac{\lambda_{2}}{\lambda_{1}}\left\{h_{1}\left[\left(h_{2}-h_{1}\right) u_{2}\right]\right\}_{x} \\
+\frac{\gamma_{2}}{\beta_{2}}\left(\alpha_{2} \frac{\beta_{1}}{\beta_{2}}-\alpha_{1}\right) \frac{\lambda_{2}}{\lambda_{1}}\left\{h_{1}\left[\left(h_{2}-h_{1}\right) u_{2}\right]\right\}_{x}+\gamma_{2} \frac{g}{\beta_{2}} \frac{\beta_{1}}{\beta_{2}} \frac{\lambda_{2}}{\lambda_{1}}\left\{h_{1}\left[\left(h_{2}-h_{1}\right) \eta_{x}\right]_{x}\right\}_{x}+\frac{1}{2} \frac{\alpha_{1}}{\beta_{2}}\left(h_{1}^{2} u_{1}\right)_{x x} \\
H_{2}\left(\mathrm{u}_{1}\right)=\frac{1}{2} \frac{\beta_{1}}{\beta_{2}}\left(h_{1}^{2} u_{1}\right){ }_{x x}
\end{gathered}
$$

The term with the subscript in Eqs. (64)-(75) implies that the term is taken derivative with respect to the subscript. The third-order Adams-Bashforth predictor scheme is used as

$$
\begin{gathered}
\eta^{n+1}=\eta^{n}+\frac{\Delta t}{12}\left(23 E^{n}-16 E^{n-1}+5 E^{n-2}\right) \\
U_{1}^{n+1}=U_{1}^{n}+\frac{\Delta t}{12}\left(23 F_{1}^{n}-16 F_{1}^{n-1}+5 F_{1}^{n-2}\right)+2 H_{1}^{n}-3 H_{1}^{n-1}+H_{1}^{n-2} \\
U_{2}^{n+1}=U_{2}^{n}+\frac{\Delta t}{12}\left(23 F_{2}^{n}-16 F_{2}^{n-1}+5 F_{2}^{n-2}\right)+2 H_{2}^{n}-3 H_{2}^{n-1}+H_{2}^{n-2}
\end{gathered}
$$

where the superscripts $n$ and $n+1$ denote the present and the future time steps, respectively. The variables $u_{i, j}^{n+1}$ and $v_{i, j}^{n+1}$ which are included in $U_{i, j}^{n+1}$ and $V_{i, j}^{n+1}$, respectively, are calculated using the LU decomposition method. After the values of $(\eta, u, v)_{i, j}^{n+1}$ are evaluated, the fourth-order AdamsMoulton corrector scheme is used as

$$
\begin{gathered}
\eta^{n+1}=\eta^{n}+\frac{\Delta t}{24}\left(9 E^{n+1}+19 E^{n}-5 E^{n-1}+E^{n-2}\right) \\
U_{1}^{n+1}=U_{1}^{n}+\frac{\Delta t}{24}\left(9 F_{1}^{n+1}+19 F_{1}^{n}-5 F_{1}^{n-1}+F_{1}^{n-2}\right)+H_{1}^{n+1}-H_{1}^{n} \\
U_{2}^{n+1}=U_{2}^{n}+\frac{\Delta t}{24}\left(9 F_{2}^{n+1}+19 F_{2}^{n}-5 F_{2}^{n-1}+F_{2}^{n-2}\right)+H_{2}^{n+1}-H_{2}^{n}
\end{gathered}
$$

The corrector step is iterated until total relative error between successive results is within a certain limit.

\section{MODEL VERIFICATION}

\section{Linear waves inside two porous layers}

The computational domains for waves propagate in 2 porous layers are given in Figs. 1 (a) and (b). The laminar and turbulent resistances and the added mass coefficient of the two porous layers are the same 
and given by $\alpha_{l}=150, \alpha_{t}=1.75, \kappa=0.47$, the porosities are $\lambda=0.44 ; 0.9$, the material sizes are $d=0.67 \mathrm{~cm} ; 3 \mathrm{~cm}$.

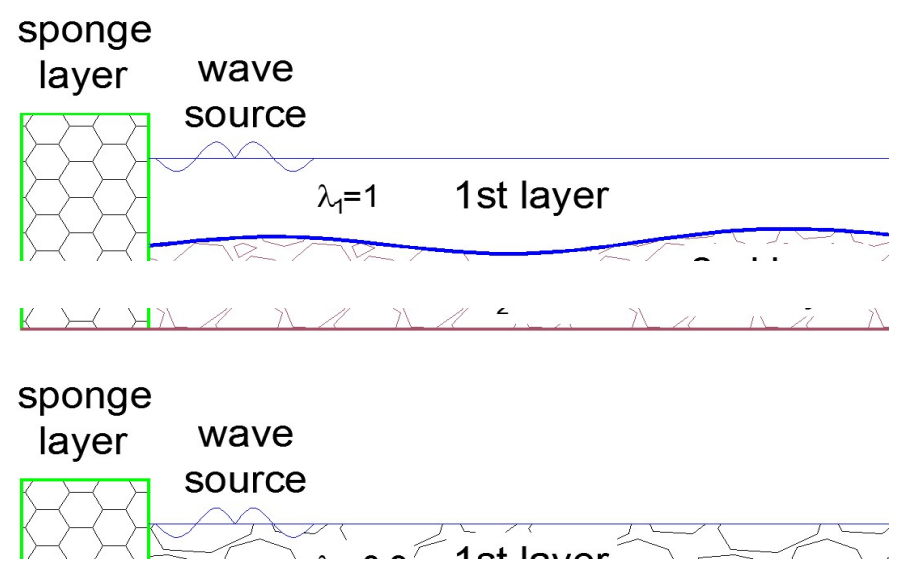

a)

b)

Figure 1. One dimensional computational domain

Figs. 2 (a), (b), and (c) compare numerical solutions of water surface elevation and wave amplitude with the corresponding analytical solutions in shallow and deep waters with different porosities. The water depth of lower layer is double the upper layer (i.e., $h_{2}=2 h_{1}$ ). In this case, the wave amplitude is negligibly small and thus, we can use the analytical solution for linear waves given by $a_{0} \exp \left(-k_{i} x\right)$ where $k_{i}$ is imaginary part of the complex wavenumber. The numerical solutions of surface elevation and wave amplitude are almost the same as the analytical solution for all the cases. In deep water with the first non-porous water layer and the second porous layer, waves propagate with almost no energy dissipation. Waves in shallow water are damped more than in deep water.

$$
k h_{1}=0.1 \pi, h_{2} / h_{1}=2, T=6 \mathrm{sec}
$$
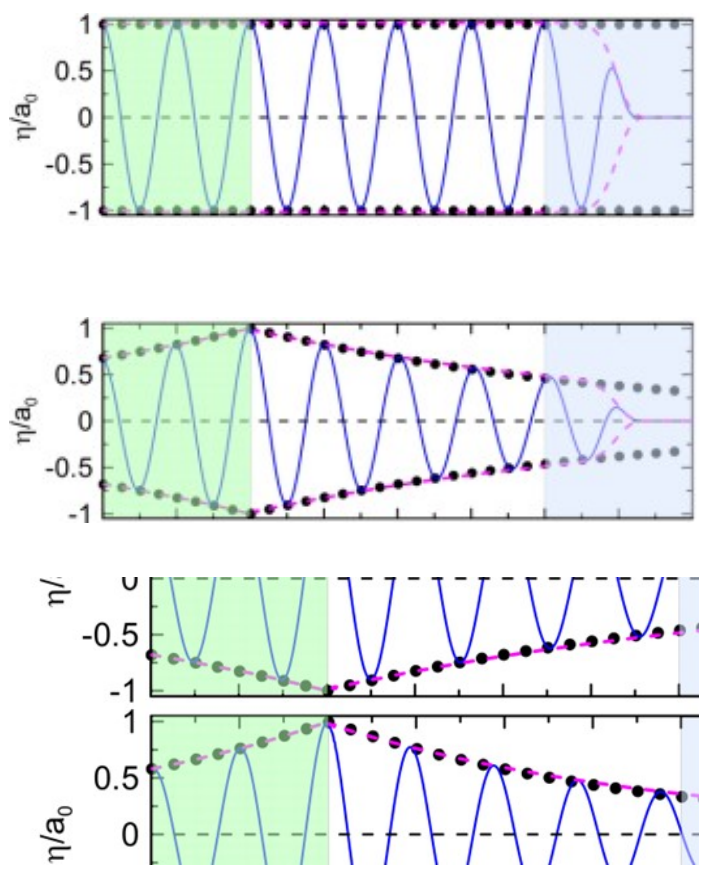

$$
k h_{1}=\pi, h_{2} / h_{1}=2, T=1.6 \mathrm{sec}
$$
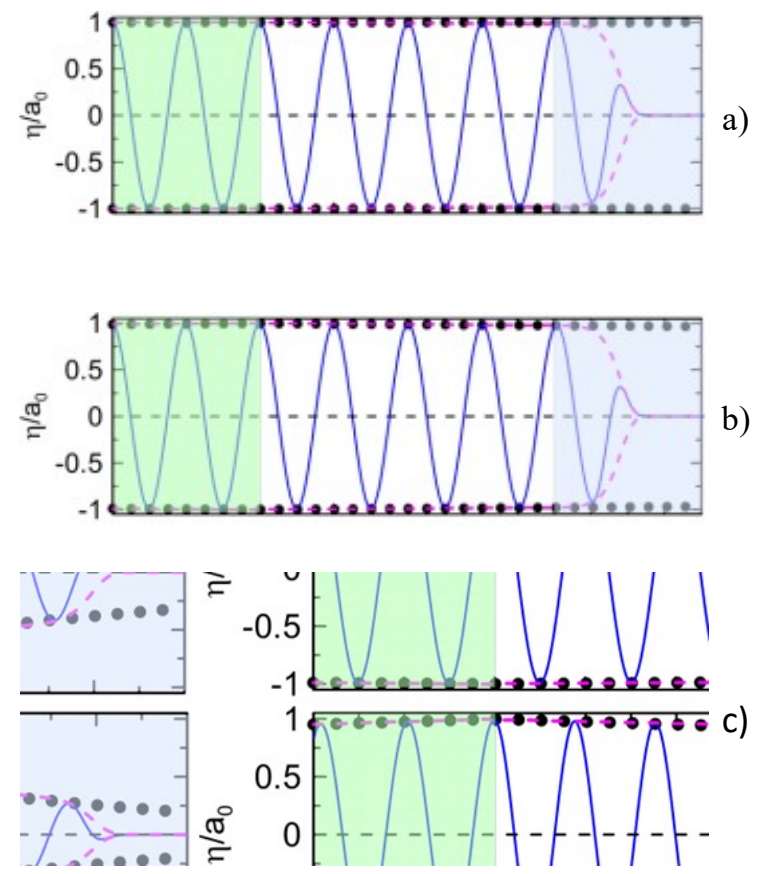

Figure 2. Normalized water surface elevation and amplitude in shallow (left figures) and deep (right figures) waters. a) $\lambda_{1}=1, \lambda_{2}=1$; b) $\lambda_{1}=1, \lambda_{2}=0.44, d_{2}=0.67 \mathrm{~cm} ;$ c) $\lambda_{1}=0.9, d_{1}=3 \mathrm{~cm}$, 
$\lambda_{2}=0.44, d_{2}=0.67 \mathrm{~cm}$ blue solid line $=$ numerical solution of water surface elevation; dashed line $=$ numerical solution of wave amplitude; filled circle $=$ exact solution of wave amplitude..

\section{Waves over a submerged triangular porous bar}

Furthermore, the numerical results of the developed model are verified with the experimental data of Hsiao et al. (2002) for nonlinear waves. The experiment was conducted for the horizontal onedimensional waves propagating over a submerged triangular porous breakwater. Waves with height of $2.7 \mathrm{~cm}$ and period of $1 \mathrm{sec}$ are internally generated in a constant depth of $0.175 \mathrm{~m}$. The porosity characteristics of the porous breakwater are given as $\lambda=0.42, \alpha_{l}=1100$, $\alpha_{t}=0.81, \kappa=0.47, \mathrm{~d}=19 \mathrm{~mm}$. The computational domain with 9 wave gauges is given in Fig. 3 .

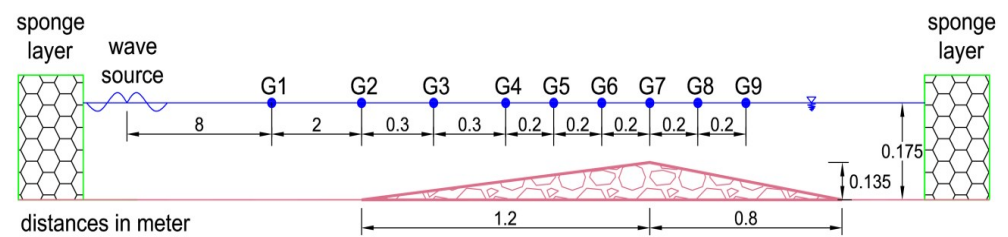

Figure 3. Computational domain given by Hsiao et al. (2002)

Fig. 4 shows that the numerical solutions of water surface elevations are close to the experimental data at all gauges. It should be noted that using the present model we do not need to use any matching conditions at the interfaces of the submerged porous breakwater. It should be noted that gauge G1 was installed in front of the toe of the submerged breakwater while gauge G7 was at the crest of the breakwater.
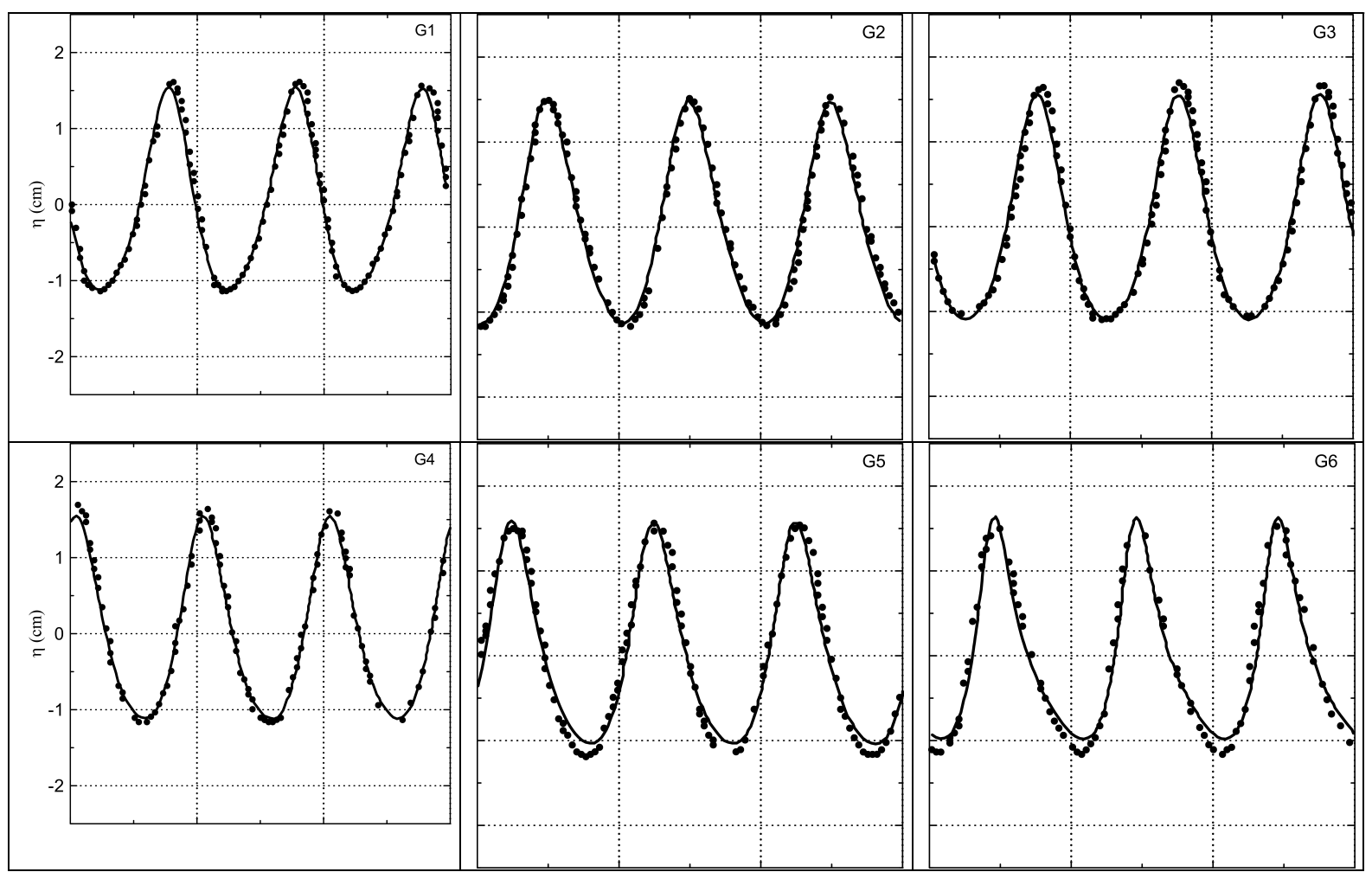


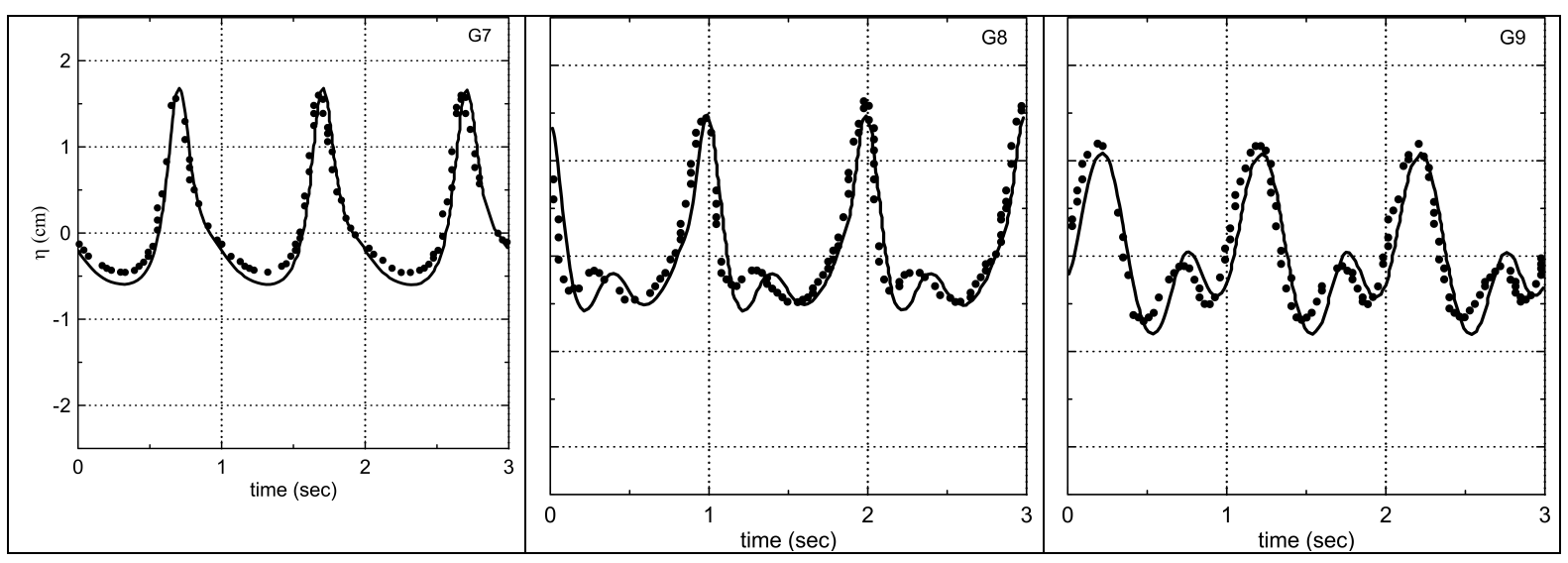

Figure 4. Comparison of present numerical solutions with experimental data of Hsiao et al. (2002): solid line $=$ numerical solution; filled circle $=$ experimental data

\section{CONCLUSIONS}

This paper derived an extended Boussinesq model for waves in two porous layers considering inertial as well as drag resistances. For linear waves in two porous layers, numerical solutions are close to analytical solutions. For waves above a submerged triangular porous breakwater, numerical solutions are well compared with the experimental data of Hsiao et al. (2002).

\section{ACKNOWLEDGMENTS}

This research was supported by Basic Science Research Program through the National Resear ch Foundation of Korea (NRF) funded by the Ministry of Education (No. NRF-2018R1D1A1 B07048606). The second author was supported by a grant (No. KH1811) from Ho Chi Minh City University of Transport.

\section{REFERENCES}

Burcharth, H.F., Andersen, O.H., 1995. On the one-dimensional steady and unsteady porous flow equations. Coastal Engineering 24, 233-257.

Cruz, E.C., Isobe, M., Watanabe, A., (1997). Boussinesq equations for wave transformation on porous beds. Coastal Engineering 30, 125-156.

Ergun, S., 1952. Fluid flow through packed columns. Chemical Engineering Progress 48, 89-94.

Forchheimer, P., 1901. Wasserbewegung durch boden. Z. Ver. Deutsch. Ing. 45, 1782-1788.

Hsiao, S.-C., Liu, P.L.-F., Chen, Y., (2002). Nonlinear water waves propagating over a permeable bed. Proc. Royal Society of London A 458, 1291-1322.

Irmay, S., 1958. On the theoretical derivation of Darcy and Forchheimer formulas. Transactions, American Geophysical Union 39, 702-707.

Liu, P.L.-F., Wen, J., 1997. Nonlinear diffusive surface waves in porous media. J. Fluid Mechanics 347,119-139.

Sollitt, C.K., Cross, R.H., 1972. Wave transmission through permeable breakwater. Proc. 13th International Conference on Coastal Engineering, ASCE 1827-1846.

Van Gent, M.R.A., 1994. The modeling of wave action on and in coastal structures. Coastal Engineering 22, 311-339.

Vu, V.N., Lee, C., Jung, TH., (2018). Extended Boussinesq equations for waves in porous media. Coastal Engineering 139, 85-97. 